\title{
Intermolecular Cooperative Exciton-Plasmon Energy Transfer Affected by Molecular Sizes of Organic Light Emitting Materials
}

\author{
K. Kasahara ${ }^{1}$, N. Ikeda ${ }^{2}$, Y. Sugimoto ${ }^{2}$ \\ ${ }^{1}$ College of Science and Engineering, Ritsumeikan University, \\ 1-1-1 Noji-higashi, Kusatsu, Shiga 525-8577, Japan \\ kasahara@se.ritsumei.ac.jp \\ ${ }^{2}$ Center, National Institute for Materials Science (NIMS), \\ 1-2-1 Sengen, Tsukuba, 305-0047, Japan \\ IKEDA.Naoki@nims.go.jp, sugimoto.yoshimasa@nims.go.jp
}

\section{Extended Abstract}

Luminous efficiencies of organic light emitting diodes are affected by surface plasmon (SP)-mediated light emission which occurs on a neighboring metallic electrode surface. Light output can be increased when light emission efficiency is low due to nonradiative recombination via impurity levels in the emission layer. In our previous experiments [1], it was found that the SP-mediated light emission characteristics could not be explained quantitatively by a physical picture that one SP was created from one exciton. Thus, we have investigated a possibility of intermolecular cooperative exciton-plasmon energy transfer. When $M$ excitons create one SP, the substantial scattering probability of the SP becomes $M$ times the probability for the case of out-of-phase condition. In this study, the ratio of photoluminescence from an organic layer (Alq 3 or NPB) formed on an Ag layer to that without the Ag layer was experimentally obtained as a function of the organic layer thickness. Together with the conversion rate of nonradiative components to radiation modes via SPs, $Y$, the $M$ value was determined so that calculated results of the ratios agreed with the experimental data.

The photoluminescence of a sample was compared with that from a reference device. The reference device was made by depositing $\mathrm{Alq}_{3}$ or NPB on a dielectric multilayer film, whose reflectivity was set to be $\sim 100 \%$ at $530 \mathrm{~nm}$ for Alq ${ }_{3}$, and $420 \mathrm{~nm}$ for NPB, respectively. Thus, the ratio of photoluminescence could be obtained without worrying about the difference of optical interference arising inside the emission layer. The proportion of nonradiative components was measured by depositing $\mathrm{Alq}_{3}$ or NPB on a frosted glass and by using an integration sphere.

The scattering rate of SPs at the wavelength was calculated from the probability obtained from the average height and period of bumps measured by AFM, and from the SP propagation distance. Although the $Y$ values of Alq 3 and NPB were approximately $10^{-3}$, the obtained results of $M$ for $\mathrm{Alq}_{3}$ and NPB were remarkably different: 32 for $\mathrm{Alq}_{3}$ and 2 for NPB. This was probably due to the size of NPB being larger than $\mathrm{Alq}_{3}$. The number of excitons with the same phase should depend on intermolecular distance. There is a possibility that enhanced photon scattering observed in random lasers [2] occurs in the devices, generating local coherence.

\section{References}

[1] Y. Mizoguchi, T. Yoneda, T. Ishiguro, K. Kasahara, N. Ikeda, and Y. Sugimoto, "Light enhancement dependence on organic film thickness caused by the generation of surface plasmons in phase and their scattering," in the 8th International Conference on Metamaterials, Photonic Crystals and Plasmonic (META2017), Songdo Convensia, Incheon-Seoul, South Korea, pp. 01-06, 2017.

[2] D.S. Wiersma, "The physics and applications of random lasers," Nat. Phys., vol. 4, pp. 359-367, 2008. 